\section{Rekombinante Revolution in der Diagnostik}

G rundlage jeder allergologischen Therapieintervention ist eine solide Diagnostik. Führen Anamnese und Hauttestung zu keinen eindeutigen Ergebnissen, bieten sich In-vitro-Untersuchungen an. Hier können durch den Einsatz von rekombinanten Allergenen zusätzliche Informationen gewonnen werden, erklärte Prof. Dr. Stefan Vieths, Langen. Rekombinant hergestellte Allergenmoleküle in hochreiner Form sind die am besten charakterisierten Reagenzien für die In-vitro-Diagnostik, sie haben eine höhere Sensitivität für den Nachweis spezifischer IgE-Antikörper als nativ hergestellte Allergenextrakte.

Vorteile bringen rekombinante Allergene z. B. in der Diagnostik von Nahrungsmittelallergien. So gelingt durch die Komponenten-aufgelöste Diagnostik eine Unterscheidung zwischen einer Sensibilisierung gegen „milde“, meist Birkenpollen-assoziierte Nahrungsmittelallergene und „aggressive“ Nahrungsmittelallergene, etwa aus der Gruppe der Lipidtransferproteine, die zu schweren
Symptomen führen können. Eine solche Unterscheidung ist mit Gesamtallergenextrakten nicht möglich. Allerdings ließen sich auch mit rekombinanten Allergenen falsch-positive Ergebnisse nicht ganz ausschließen, warnte Vieths.

Detaillierte Informationen über das Sensibilisierungsprofil des Patienten

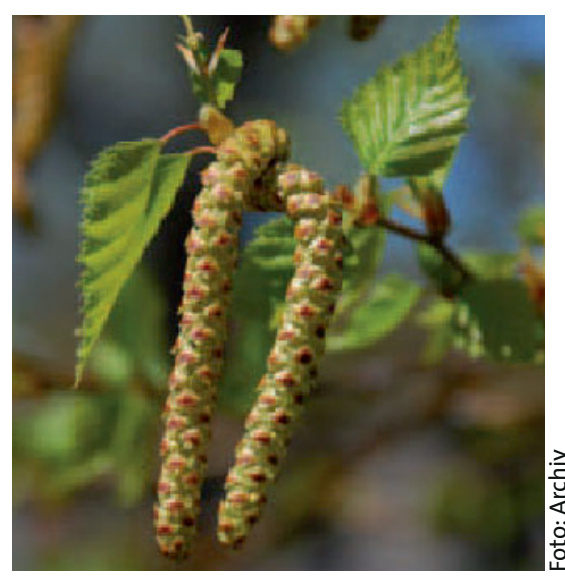

Löst Bet $v 1$ die Symptome aus oder Bet v 2? Die Diagnostik mit rekombinanten Allergenen kennt die Antwort. sind auch bei der Entscheidung für oder gegen eine Hyposensibilisierung hilfreich, erläuterte Priv.-Doz. Dr. Vera Mahler, Erlangen. So gibt es „Birkenpollenallergiker", die lediglich eine Sensibilisierung gegen hoch kreuzreaktive Minorallergene wie Profilin aufweisen. Bei diesen Patienten führt eine Immuntherapie mit Birkenpollenextrakten möglicherweise zu keinem guten Ergebnis. Bei einer Diagnostik mit nativen Allergenen würde ein solcher Patient allerdings als Birkenpollenallergiker identifiziert. Erst in der differenzierten, Komponenten-aufgelösten Diagnostik weist ein negativer rBet-v-1-Nachweis zusammen mit einem positiven rBet-v2,4-Nachweis auf die Profilin-Sensibilisierung hin. Eine Immuntherapie wäre dann allenfalls mit Präparaten sinnvoll, die auch auf Profilin als Minorallergen standardisiert sind.

Symposium „Rekombinante Allergene in der Diagnostik - klinische Einsatzmöglichkeiten“, unterstützt von Phadia, Freiburg. Zweiter Gemeinsamer Deutscher Allergiekongress, Lübeck, 26.-29. September 2007

\title{
Neue Therapiechancen konsequent nutzen
}

$\mathrm{B}$ ei der spezifischen Immuntherapie mit konventionellen Aufdosierungsschemata erfolgt die Applikation des Allergens meist wöchentlich über mehrere Monate. Eine klinisch wirksame Gesamtdosis wird dabei sehr spät erreicht. Prof. Dr. Ludger Klimek, Wiesbaden, verwies auf die Vorteile von chemisch modifizierten Allergoiden wie Depigoid ${ }^{\circledR}$ : Hier bleibt die Antigenpräsentation gegenüber T-Zellen mit der gewünschten Immunmodulation erhalten, während die denaturierten und polymerisierten Allergene eine extrem niedrige IgE-Bindungskapazität aufweisen. Damit ist eine sichere Aufdosierung in kürzester Zeit möglich. Klimek verwies auf die Arbeit von Casanovas et al. (Int Arch Allergy Immunol 2006; 139: 153-8): In dieser Untersuchung hatten 1.068 Patienten eine Quick-Aufdosierung erhalten, die mit nur zwei Injektionen an einem Tag bereits abgeschlossen war. Schwere $\mathrm{Ne}$ benwirkungen wurden überhaupt nicht beobachtet, leichte systemische Nebenwirkungen traten nur bei acht Patienten auf.

Prof. Dr. Eckard Hamelmann, Berlin, machte auf die enge Assoziation zwischen Asthma und Allergien aufmerksam: Zwei Drittel aller Asthmatiker sind allergisch und über 50\% der Patienten mit schwerem Asthma bronchiale leiden unter Allergien. Speziell hier bietet sich das Therapiekonzept mit dem monoklonalen Anti-IgE-Antikörper Omalizumab (Xolair ${ }^{\circledR}$ ) an. Das Medikament bindet freies IgE und auch das zellgebundene $\operatorname{IgE}$ wird reduziert. Zusätzlich kommt es zu einer Herabregulation der hochaffinen IgE-Rezeptoren. Die klinische Wirksamkeit von Omalizumab konnten Bousqet et al. (Allergy 2005; 60: 302-8) eindrucksvoll mit einer signifikanten Reduktion der
Zahl der Asthmaexazerbationen um 38\% belegen. Die schweren Exazerbationen gingen sogar um $57 \%$ zurück.

Dass der Behandlungserfolg der spezifischen Immuntherapie durch die gleichzeitige Anwendung von Omalizumab gesteigert werden kann, zeigte Priv.-Doz. Dr. Matthias Kopp, Freiburg. Studien bei Kindern wie auch bei Erwachsenen belegen den additiven Effekt beider Therapien. Sowohl die Symptomlast als auch die Rate schwerer Nebenwirkungen nimmt unter dieser Kombinationstherapie im Vergleich zur alleinigen Immuntherapie ab. Diese duale Therapie könnte in Zukunft die Effektivität und Sicherheit der Behandlung allergischer Patienten noch weiter steigern.

Symposium „Synergien zwischen AntiIgE und SIT - eine Vision?", unterstützt von Novartis, Nürnberg. Zweiter Gemeinsamer Deutscher Allergiekongress, Lübeck, 26.-29. September 2007 